Rabaska

Revue d'ethnologie de l'Amérique française

\title{
Chaire de recherche du Canada sur l'identité métisse
}

Denis Gagnon

Volume 6, 2008

URI : https://id.erudit.org/iderudit/000182ar

DOI : https://doi.org/10.7202/000182ar

Aller au sommaire du numéro

Éditeur(s)

Société québécoise d'ethnologie

ISSN

1703-7433 (imprimé)

1916-7350 (numérique)

Découvrir la revue

Citer ce document

Gagnon, D. (2008). Chaire de recherche du Canada sur l'identité métisse.

Rabaska, 6, 269-271. https://doi.org/10.7202/000182ar d'utilisation que vous pouvez consulter en ligne.

https://apropos.erudit.org/fr/usagers/politique-dutilisation/ 


\section{Ouest}

\section{Chaire de recherche du Canada sur l'identité métisse}

Collège universitaire de Saint-Boniface

200, avenue de la Cathédrale Téléphone : (204) 237-1818, poste 454

Saint-Boniface (Manitoba) R2H 0H7 Courriel : degagnon@ustboniface.mb.ca

\section{Faits saillants}

Les événements marquants de l'année 2007-2008 touchent le recrutement d'étudiants diplômés (Saskatchewan, Québec, France) ; la codirection de deux thèses de doctorat et d'un mémoire de maîtrise (Manitoba, Québec, France) ; le développement d'un cadre théorique, conceptuel et méthodologique appliquée aux études métisses ; et la diffusion des données aux plans national et international. Ces progrès ont permis d'explorer l'identité métisse au Québec, dans les Maritimes et au Labrador dans une perspective comparative avec les Métis de l'Ouest ; d'offrir des occasions de terrains anthropologiques à des doctorants ; de favoriser le réseautage de la chaire au Canada (Manitoba, Saskatchewan, Québec, Terre-Neuve-Labrador) et en France (Valenciennes, Rouen, Paris I, ÉHÉss) par le biais de nombreuses conférences publiques, de séminaires et de communications dans le cadre d'ateliers et de colloques auxquels ont participé plusieurs assistants de recherche.

La chaire est devenue le noyau canadien de recherche et de formation en anthropologie du métissage, un champ jusqu'alors inexistant au Canada. Grâce au développement d'un cadre théorique basé sur l'étude des relations de pouvoir, nos travaux ont démontré non seulement la complexité des multiples niveaux des identités métisses au Canada et dans les anciennes colonies européennes, mais aussi la complexité des typologies utilisées pour définir cette identité qui découle de deux approches qui semblent irréconciliables : les approches exclusives et inclusives. L'approche exclusive se base sur les critères établis par les Métis de l'Ouest canadien qui ne reconnaissent cette identité qu'aux individus descendants des Métis de la rivière Rouge (les « vrais » Métis), et sur les critères juridiques de l'Arrêt Powley (2003), excluant ainsi tous les « autres » Métis canadiens à l'exception des Métis de l'Ontario. L'approche inclusive se base sur l'article 32 de la Constitution (1982). Elle met l'accent sur la reconnaissance de communautés métisses historiques et elle s'applique à tous les Métis du Canada. Appelée à réaliser des enquêtes visant à prouver l'existence de communautés métisses par le gouvernement et par ces communautés, la chaire a réussi à conserver sa neutralité et son indépendance dans un contexte de recherche hautement 
judiciarisé en ne réalisant que des enquêtes ethnographiques et des recherches sur l'identité. Nous devons demeurer extrêmement prudents dans nos interventions publiques et gouvernementales, et ne pas participer à la définition d'une identité métisse, car notre position peut être interprétée défavorablement par les tenants d'une des deux approches. Une constatation très importante est que le plus grand danger pour ces communautés demeure la reconnaissance juridique de leurs droits autochtones qui pourrait signifier, à moyen terme, l'extinction de cette identité par le métissage, car les Métis qui se métisseront au-delà de deux générations perdront leur statut.

\section{Diffusion}

Concernant la diffusion des données, un des événements les plus importants de l'année 2007-2008 a été la publication de Statistiques Canada: Peuples autochtones du Canada en 2006, qui montre que le nombre de Canadiens s'identifiant comme Métis a quadruplé depuis 1991. J'ai participé à une entrevue télévisée, une entrevue radiophonique et un article de journal pour commenter ce rapport et, grâce aux travaux entrepris depuis 2004, nous avons pu contextualiser et fournir aux médias des explications sur ce phénomène difficilement explicable. J'ai accordé une entrevue à la chaîne de télévision APTN lors d'une conférence publique à Saskatoon ; un article de vulgarisation scientifique est paru dans la revue Découvrir International ; et j'ai accordé une entrevue à un écrivain pour un article sur les Métis à paraître dans Canadian Geographic. Je suis également sollicité par courriel pour donner mon avis sur certains événements et sur certaines circonstances touchant l'identité métisse. L'équipe de recherche est aussi en contact étroit avec plusieurs communautés métisses canadiennes et leur présente les objectifs et la méthodologie de nos recherches.

\section{Collaborations}

Au sujet des collaborations, je collabore avec Léonard Rivard dans le cadre de la subvention de l'Aruc sur les identités francophones de l'Ouest au Collège universitaire de Saint-Boniface (CusB). Le projet de publication des actes du colloque Gabriel-Dumont en codirection avec Lise Gaboury-Diallo (CusB) et Denis Combet (Brandon) a demandé une étroite collaboration tout au cours de l'année. Je collabore également avec plusieurs professeurs d'universités canadiennes : Jean Guy Meunier (UQÀM) et Robert Papen (UQÀM), avec Laurier Turgeon (Laval) par le biais de Martin Fournier dans le cadre du Dictionnaire du patrimoine culturel de l'Amérique française, et une nouvelle collaboration a débuté le 31 mars avec Luc Noppen (UQÀM) dans le cadre du Forum canadien de recherche publique sur le patrimoine. J'ai également collaboré avec Danielle Cyr (York) pour une demande de subvention au CRSH. 
Je suis membre régulier du Centre interuniversitaire d'études et de recherches autochtones (CIÉRA) de l'Université Laval avec lequel nous projetons de développer un volet de recherche sur les communautés métisses. La collaboration avec des chercheurs français a été très fructueuse. Invité à donner une série de conférences et de séminaires en France (Valenciennes, Rouen, Paris I, ÉHÉss), j'ai poursuivi la consolidation de ce réseautage en organisant un atelier multidisciplinaire sur le métissage qui aura lieu en juin 2008 et réunira plus de quinze chercheurs de pointe et professeurs d'universités françaises au Centre de recherche d'histoire nord-américaine (CRHNA) à l’Université Paris I (Panthéon-Sorbonne).

\section{Recherche}

L'année 2007-2008 a été particulièrement intéressante au plan de la recherche. Je suis cochercheur pour quatre projets, dont trois du CRSH. Il s'agit du projet d'Analyse thématique de documents textuels dirigé par Jean-Guy Meunier (UQÀM); de l'Alliance de recherche université communautés sur les identités francophones de l'Ouest, dirigé par Léonard Rivard (CusB), dont je suis responsable du volet métis, et du projet de Forum canadien de recherche publique sur le patrimoine, dirigé par Luc Noppen (UQÀM), amorcé le 31 mars 2008. Je suis également cochercheur pour le Dictionnaire du patrimoine culturel de l'Amérique française, dirigé par Laurier Turgeon (Laval) et financé par le SAIC (Québec). J'ai également participé en tant que cochercheur à deux demandes de subventions au CRSH, dont une a été acceptée en avril 2008 (avec Jean-Guy Meunier de l’UQÀM).

Denis Gagnon

\section{Société historique de Saint-Boniface}

Centre du patrimoine

340, boulevard Provencher

Saint-Boniface (Manitoba) R2H 0G7

Téléphone : (204) 233-4888

Télécopieur : (204) 231-2562

Courriel : shsb@shsb.mb.ca

Toile : www.shsb.mb.ca

Aperçu de l'année 2007-2008

Le conseil, le personnel et nos bénévoles ont contribué à réaliser la mission de la SHSB cette année. Ainsi, nous avons travaillé à acquérir, à conserver et à mettre en valeur le patrimoine francophone et métis de l'Ouest canadien et en particulier du Manitoba. Pour ce faire, nous avons revu notre plan 